\title{
Employing Parasite Against Cancer: A Lesson From the Canine Tapeworm Echinococcus Granulocus
}

\begin{abstract}
Wang Guan ${ }^{1 t}$, Xiaoqin Zhang ${ }^{2 \dagger}$, Xiao Wang ${ }^{1 *}$, Shun Lu $^{3}$, Jun Yin ${ }^{3}$ and Jinxin Zhang ${ }^{4 *}$
${ }^{1}$ Department of Cancer Prevention and Treatment, Sichuan Cancer Hospital and Institute, Sichuan Cancer Center, School of Medicine, University of Electronic Science and Technology of China, Chengdu, China, ${ }^{2}$ Intensive Care Unit, Sichuan Provincial People's Hospital, School of Medicine, University of Electronic Science and Technology of China, Chengdu, China, ${ }^{3}$ Radiation Oncology Key Laboratory of Sichuan Province, Sichuan Cancer Hospital and Institute, Sichuan Cancer Center, School of Medicine, University of Electronic Science and Technology of China, Chengdu, China, ${ }^{4}$ School of Public Health, Sun Yat-sen University, Guangzhou, China
\end{abstract}

\section{OPEN ACCESS}

Edited by:

Salvatore Salomone,

University of Catania, Italy

Reviewed by:

Hossein Yousofi Darani, Isfahan University of Medical

Sciences, Iran

Ben Roche,

Institut de recherche pour le développement (IRD), France

*Correspondence:

Wang Xiao

wangxiaogz@163.com

Zhang Jinxin

zhjinx@mail.sysu.edu.cn

tThese authors have contributed equally to this work

Specialty section:

This article was submitted to Experimental Pharmacology and Drug Discovery, a section of the journal

Frontiers in Pharmacology

Received: 24 June 2019 Accepted: 03 September 2019 Published: 25 September 2019

Citation:

Guan W, Zhang X, Wang X, Lu S, Yin J and Zhang J (2019) Employing Parasite Against Cancer: A Lesson

From the Canine Tapeworm Echinococcus Granulocus.

Front. Pharmacol. 10:1137. doi: 10.3389/fphar.2019.01137
Cystic echinococcosis (CE), a devastating zoonotic condition caused by the tapeworm Echinococcus granulosus, remain a significant public health problem worldwide. However, after a negative correlation between solid tumor and CE has been incidentally discovered, accumulating evidence have suggested that this parasite may induce anticancer effect through activating host immune response and secreting molecules with anticancer potential, which may provide some new understanding for immunotherapy. This article will review the evidence supporting the anticancer effect of $E$. granulosus and its underlying mechanisms and discuss the possible implications in immunotherapy.

Keywords: Echinococcus granulosus, cancer, immunotherapy, immune response, adjuvant

\section{INTRODUCTION}

With a new era of immuno-oncology on the horizon, numerous cancer patients have benefited from the great advances in immunotherapy, especially the discovery of immune checkpoint inhibitors (ICIs), such as cytotoxic T lymphocyte-associated protein 4, programmed cell death 1 , and programmed cell death ligand 1 inhibitors (Darvin et al., 2018). However, the great advantages of these immunotherapeutic strategies are also hampered by major limitations, which restrict them from benefiting a broader range of cancer patients. For example, only $20 \%$ of patients with advanced carcinoma (except Hodgkin's lymphoma and melanoma) would benefit from programmed cell death 1 or programmed cell death ligand 1 inhibitors (Balar and Weber, 2017). Similarly, the clinical response rate is very low even in patients receiving anti-CTLA4 therapy for unresectable or metastatic melanoma (the first approved indication of anti-CTLA4 therapy) (Savoia et al., 2016). Meanwhile, patients receiving ICIs, whether in single drug or in combination, would experience unique immune-related adverse events, ranging from mild adverse effect to potentially lifethreatening events (Champiat et al., 2015). Hence, immunotherapeutic strategies employing other potential mechanisms are worthy of further exploring.

Echinococcus granulosus, a canine tapeworm responsible for zoonotic cystic echinococcosis (CE; also termed hydatid disease), has haunted human kind for centuries (Wen et al., 2019). E. granulosus is still highly epidemic in South America, Mediterranean countries, eastern Africa, Central Asia, and Northwestern China, with human incidence as high as 50 per 100,000 person-years according to a report from the World Health Organization (Brunetti et al., 2010). However, after the astonishingly low incidence of CE was incidentally found in patients with solid tumor in Turkey (Akgül et al., 
2003), accumulating evidence have suggested that E. granulosus may exhibit anticancer effect through host immune system, which may be employed as an immunotherapeutic strategy against cancer (Ranasinghe and McManus, 2018).

\section{E. Granulosus Against Cancer: Incidental Findings to Accumulating Evidences}

Various helminths have been proven to be a carcinogenic agent in human, such as the liver fluke Clonorchis sinensis and Opisthorchis viverini (causative agents of cholangiocarcinoma) (Feng and Cheng, 2017) and the blood fluke Schistosoma japonicum (wellknown risk factor for liver carcinoma and colorectal cancer) (Takemura et al., 1998; Hamid, 2019), Schistosoma mansoni (suspected risk factor for colorectal cancer) (Almeida et al., 2017), and Schistosoma haematobium (which leads to bladder cancer) (Berry et al., 2017). On the other hand, accumulating evidence suggested that certain helminth infection could induce anticancer activities, such as the pork worm Trichinella spiralis, which can protect infected mice against tumor growth and metastasis (Kang et al., 2013).

Coexistence of E. granulosus infection and cancer has been broadly reported, such as coexistence with hepatocellular carcinoma (Bourne and Williams, 1963; Zöld et al., 2005; Li et al., 2015), lung carcinosarcoma (Misthos et al., 2012), liver mucinous cystadenoma (Muralidhar et al., 2018), renal sarcoma (Benchekroun et al., 1979), renal adenocarcinoma (Miñana López et al., 1994), or ovarian epithelial tumor and lymphoepithelioma-like gastric carcinoma (Gungor et al., 2011). The exact relationship between E. granulosus and cancer, however, has long been unclear until the last decade, when an epidemiological study on patients with CE occasionally found a negative correlation between CE and solid tumors (Akgül et al., 2003). This extraordinary phenomenon, although still in debate (Oikonomopoulou et al., 2016), has led to a hypothesis that infection of E. granulosus may elicit protective effect against cancer. Subsequently, results from studies carried out by different research groups have supported this hypothesis. It has been demonstrated that, for example, protoscolices in hydatid cyst (the larval stage of E. granulosus) could induce cell death in WEHI164 fibrosarcoma cell in vitro (Darani et al., 2012). Moreover, vaccination with hydatid fluid induced tumor regression in a mouse model with experimental CT26 colon cancer (Berriel et al., 2013). In addition, simultaneously injection of alive protoscolices and melanoma cell could result in significantly reduced tumor growth compared with control group in a mouse model (Chookami et al., 2014). Similarly, intraperitoneal hydatidosis established by infection of protoscolices could inhibit DMBA-induced mammary tumorigenesis in rats (Altun et al., 2015). As an indirect evidence, sera from patients with hydatid disease was cytotoxic to lung cancer cell line but had no effect on fibroblast cells (Karadayi et al., 2013). Collectively, these evidences suggested that E. granulosus may elicit protective effect against certain cancer types in vitro and in vivo.

\section{Mechanisms Underlying Effect Induced by E. Granulosus}

Despite the great efforts put by researchers, little is known about the mechanisms underlying the anticancer effect induced by $E$. granulosus. Several potential mechanisms have been proposed, including direct anticancer effect induced by parasite molecules and indirect anticancer effect through activation of host immune response (Figures $\mathbf{1}$ and 2 ).

\section{Direct Anticancer Effect Induced by Parasite Molecules}

Several studies have suggested that E. granulosus could kill cancer cell or inhibit tumor growth. As noted above, protoscolex

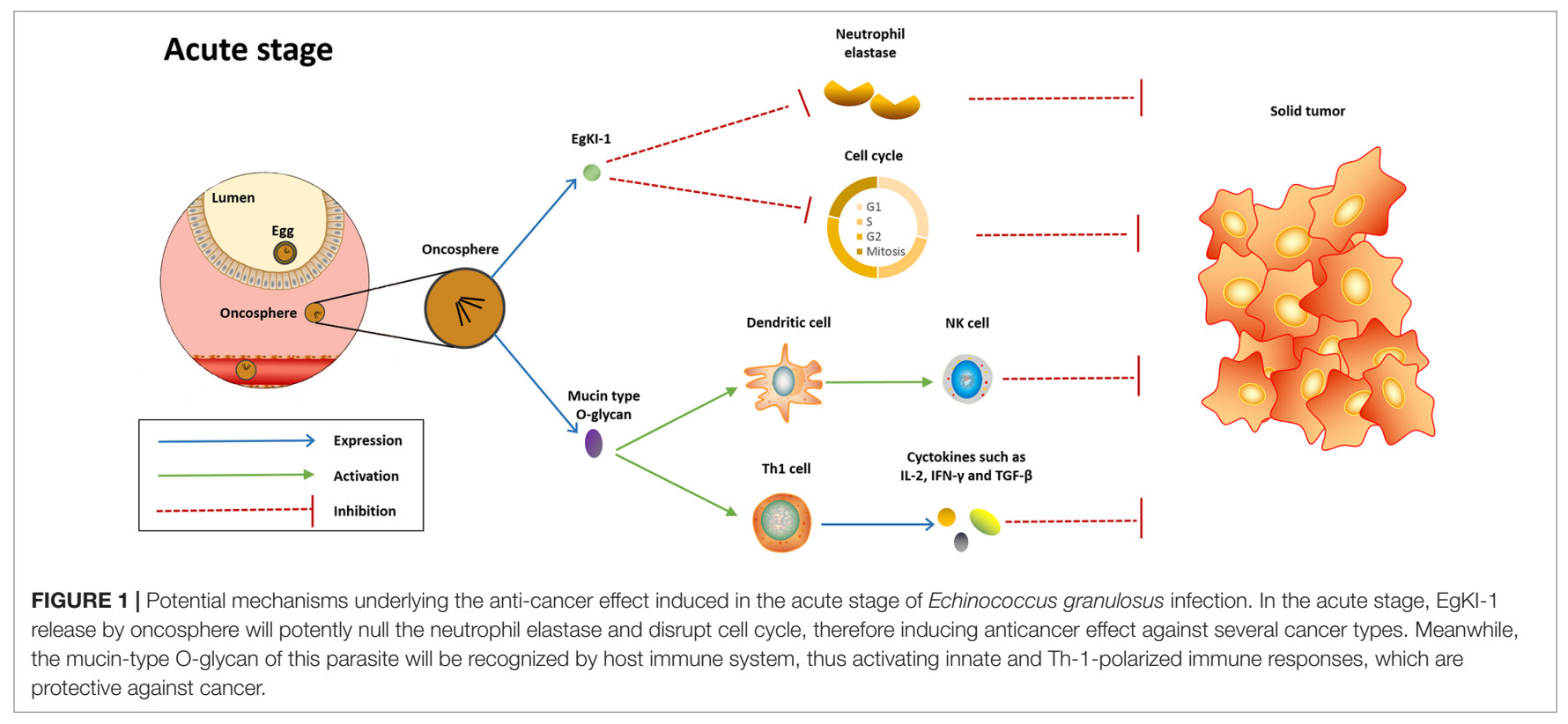




\section{Chronic stage}

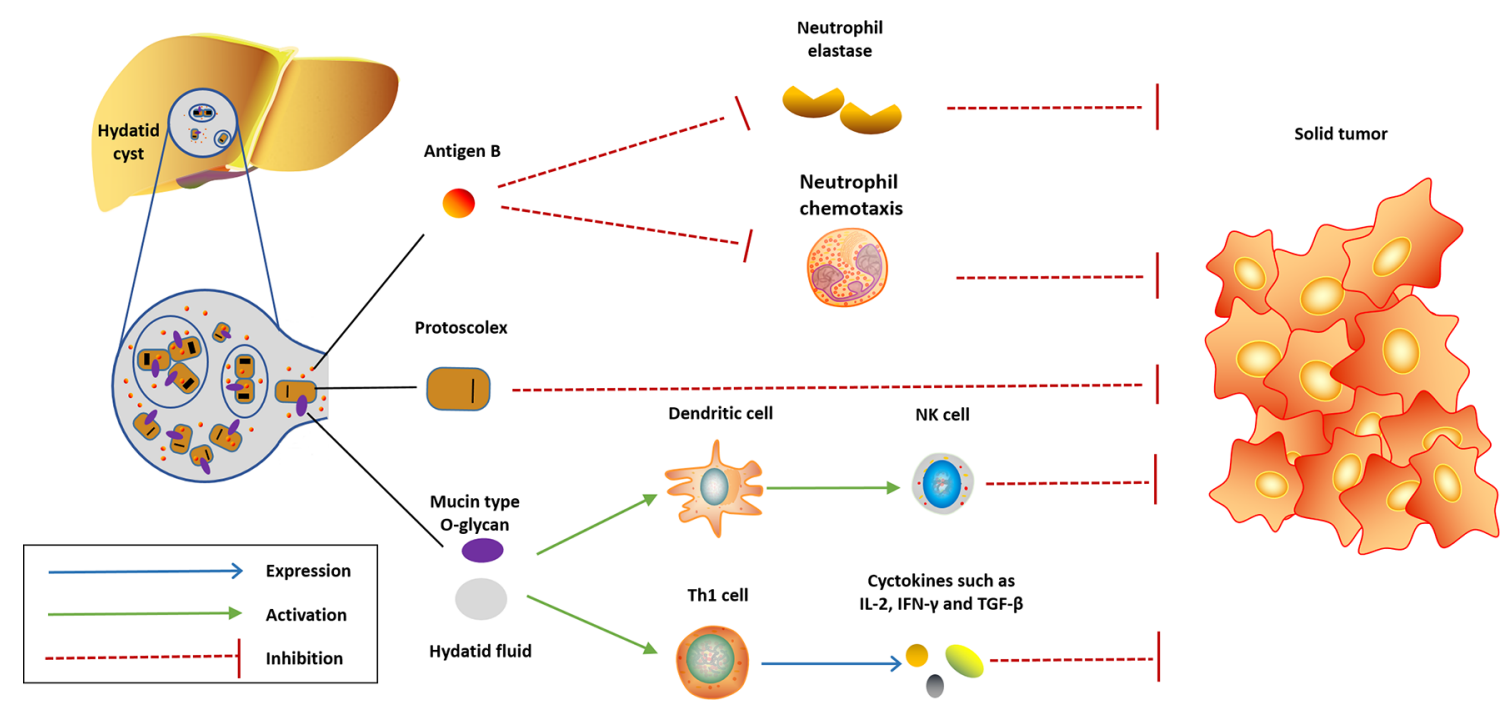

FIGURE 2 | Potential mechanisms underlying the anticancer effect induced in the chronic stage of Echinococcus granulosus infection. In the chronic stage, the content of hydatid cyst will be released into liver or other infection sites when the cyst dies or ruptures. Highly immunogenic content of hydatid cyst will quickly activate innate immunity and convert Th-2 response to Th-1 response, which are protective against cancer. AgB, another potent neutrophil elastase inhibitor highly expressed in the hydatid cyst, may exhibit anticancer effect through inhibition of neutrophil elastase and neutrophil chemotaxis. Meanwhile, the protoscolex may also play a role in the anticancer effect.

could induce cell death in WEHI-164 fibrosarcoma cell in vitro (Darani et al., 2012) and inhibit melanoma tumor growth in vivo (Chookami et al., 2014). The specific molecules involved in this process, however, remain largely unclear. Notably, a study found that treatment with different hydatid molecules, especially the protoscolex excretory/secretory (ES) molecules, could increase the number of dead cells and/or decrease the number of alive cells in HeLa cell culture; meanwhile, treatment with the same molecules neither increase the number of dead cells nor decrease the number of alive cells in vero cell culture (Aref et al., 2013). Subsequently, another study found that injection of hydatid fluid (peritoneal or tumor margin) could decrease melanoma tumor size in C57 mouse model (Darani et al., 2016). However, the alum-based adjuvant employed in the latter study is potentially confounding (Ranasinghe and McManus, 2018), since alum may induce anticancer effect through selectively stimulation of Th2 immune response (Didierlaurent et al., 2009). Recently, a research group found that the Kunitz-type protease inhibitor EgKI-1, a potent chymotrypsin and neutrophil elastase inhibitor highly expressed by oncosphere of E. granulosus (Ranasinghe et al., 2015), could inhibit several human cancers from growth and migration, probably through disrupting cell cycle and inducing apoptosis of cancer cells, without affecting normal cell growth in vitro (Ranasinghe et al., 2018). Meanwhile, EgKI-1 treatment could restrict experimental triple negative breast cancer growth in a BABL/c mouse model. The specific mechanism underlying this effect still demands further exploring. A possible explanation is that EgKI-1 may decrease cancer cell migration via potent inhibition of neutrophil elastase, which plays a pivotal role in cancer metastasis (Zhang et al., 2016). Being another potent neutrophil elastase inhibitor highly expressed in the hydatid cyst, it has been hypothesized that Antigen $\mathrm{B}(\mathrm{AgB})$ may be responsible for the anticancer effect induced during chronic state of infection. Recently, another study explored the anticancer potential of several hydatid fluid antigens, including $\mathrm{AgB}$, glycolipid, glycoprotein, and $78 \mathrm{kDa}$ fractions in breast cancer cell lines. Compared with the other molecules, less apoptosis was observed after treatment with AgB fraction (Daneshpour et al., 2019). Therefore, the possible role of $\mathrm{AgB}$ requires further exploring. In general, available evidence suggested that the molecules from the hydatid cyst, especially the ES molecules, are potentially responsible for the anticancer effect of this parasite.

\section{Indirect Anticancer Effect Through Activation of Host Immune System}

As noted above, anticancer effect has been seen in various parasites infection. Similar with parasites infection, cancer cells could also trigger innate and adaptive immunity in the process of development (Trinchieri, 2015). Therefore, it has been hypothesized that parasites may induce indirect anticancer activities via boosting host immune system when chronically infected at a low density (Darani and Yousefi, 2012), i.e., parasites infection may play as negative regulator of cancer through reactivation of host immune system (Callejas et al., 2018).

In favor of this hypothesis, antigen similarity between several parasites and certain cancer types has been reported, 
especially the cancer-associated mucin-type O-glycans (Osinaga, 2007), thus allowing crosstalk between parasites and carcinomas to ensue. For example, S. mansoni can express the Tn antigen (Nyame et al., 1987), which is associated with many cancer types (including bladder, cervix, colon, ovary, gastric, lung, and prostate cancer) and plays an important role in cancer metastasis and evasion from immunosurveillance (Fu et al., 2016). Meanwhile, the Tk antigen, which is strongly expressed at the surface of a significant proportion of human colorectal carcinomas (Meichenin et al., 2000), has been detected in Taenia hydatigena, Taenia crassiceps, and Mesocestoides vogae (Ubillos et al., 2007). In the case of E. granulosus, the initial evidence of common antigen shared with cancers came from a report published in late 1970s, which found that an immunoelectrophoresis test with hydatid fluid and serum from a patient with pulmonary carcinoma resulted in an intense precipitin band (Yong et al., 1979). Thereafter, different research groups have independently reported the antigen similarity between E. granulosus and several cancer types, especially the mucin-type O-glycans. As an example, cancer-associated Tn antigen and Sialyl-Tn (sTn) antigen have been detected in both larva and adult worm extracts and in sera from patients with hydatid disease (Alvarez Errico et al., 2001). In addition, TF antigen has been characterized in adult worm (Casaravilla et al., 2003). Besides, common antigens other than mucin-type $O$-glycans have also been detected. For example, a nonglycosylated $27 \mathrm{kDa}$ molecule was shared by human breast cancer and E. granulosus (Sharafi et al., 2016). Similarly, the hot shock protein 70 of E. granulosus displayed $60 \%$ homology with the mortalin of CT26 colon cancer cell (Berriel et al., 2013). Moreover, it has been demonstrated that the common antigens shared by E. granulosus and cancers could induce immunological cross-reaction. For example, it has been demonstrated that antigens from hydatid cyst could react with sera from patients with breast cancer, while antisera raised against laminated and germinal layers of hydatid cyst could react with ES products of cancer cell (Daneshpour et al., 2016). In addition, a 40-kDa antigen from hydatid fluid could react with sera from patients with breast cancer, while monoclonal antibody raised against this $40-\mathrm{kDa}$ antigen could react with breast cancer cell (but cell growth was unaffected) (Sharafi et al., 2017). Altogether, it is reasonable to hypothesize that E. granulosus may exhibit anticancer effect through adaptive immunity induced by common antigens.

Previous studies have demonstrated that both cellular immunity and humoral immunity induced by protozoan parasite Trypanosoma cruzi are protective against several cancer types in mice model (Kallinikova et al., 2008; Zenina et al., 2008; Zhigunova et al., 2013). In the case of $E$. granulosus, however, it is yet unclear which kind of immunity is more important in the anticancer effect. In the paradigm of onco-immunology, it has been recognized that Th1polarized response plays pivotal role in killing cancer cell and inhibiting tumor growth, whereas Th2-polarized response promotes tumor progression and metastasis (Shurin et al., 1999; Zamarron and Chen, 2011). As for E. granulosus, the immunity induced by parasite varies at different infection stages: 1) During oncosphere invasion, a Th1-polarized response will dominate; 2) in the process of cyst formation and growth, a Th2-polarized response will gradually take in charge; 3) when cyst ruptures or dies, the Th2-polarized response will be rapidly taken over by Th1-polarized response (Zhang et al., 2008; Gottstein et al., 2017). Therefore, it has been hypothesized that the anticancer effect may come from the Th1-polarized response induced at specific stages of infection ( $\mathrm{Tez}$ and $\mathrm{Tez}, 2015$ ). In addition, this may provide an explanation for the contradictive results from some epidemiological investigation (Oikonomopoulou et al., 2016) and laboratory research (Turhan et al., 2015), which showed that CE may promote cancer development and progression.

There are some evidence that support the role of humoral immunity in the anticancer effect induced by E. granulosus. As noted above, sera from patients with hydatid disease was cytotoxic to lung cancer cell line but had no effect on fibroblast cells (Karadayi et al., 2013). However, the humoral immunity seems unimportant in the anticancer effect induced by $E$. granulosus in subsequent studies. As an example, antibody against Tn-like peptide from $E$. granulosus scarcely reacted with mammary adenocarcinoma cell line $\mathrm{TA} 3 / \mathrm{Ha}$ and pancreatic adenocarcinoma cell line Panc02 (both of which strongly express Tn antigen), despite the peptide induced high level of antibody production; meanwhile, high level of interferon gamma (IFN- $\gamma$ ) was detected in mice immunized with this peptide, while interleukin (IL)-5 and IL-17 were not detected, indicating a Th1-dominant response (Noya et al., 2013). Similarly, immunization of mice bearing melanoma cancer with hydatid cyst antigens resulted in inhibition of tumor growth and IFN- $\gamma$ production (Rostami et al., 2018). Moreover, the monoclonal antibody raised against the $40-\mathrm{kDa}$ antigen hydatid fluid mentioned above could not restrict breast cancer cell growth in vitro, even though it could react with breast cancer cell (Sharafi et al., 2017). Recently, a study found that passive transfer of antisera against hydatid fluid, protoscolex antigen, or cyst wall antigen did not affect melanoma tumor growth in recipient mice that had already been challenged by melanoma cells (Darani et al., 2018). On the other hand, passive transfer of spleen cells from mice immunized with hydatid cyst, hydatid fluid, or protoscolex to recipient mice would lead to significant reduction in melanoma tumor size and tumor growth rate (Ramazninia et al., 2016; Darani et al., 2018). Therefore, Th1polarized response are more likely to be responsible for the anticancer effect induced by E. granulosus.

Besides adaptive immunity, the study noted above also detected high level of natural killer cell activation, thus indicating a role of innate immunity in the anticancer effect (Noya et al., 2013). However, a definite conclusion cannot be reached in the current stage due to lack of evidence.

In summary, E. granulosus may induce anticancer effect through direct anticancer effect, i.e., secreting various molecules with cancer killing effect, and indirect anticancer effect, i.e. activating host immune response (Th1-polarized response in particular). However, more efforts are required to shed further light on the mechanisms underlying this extraordinary effect. 


\section{Implication in Cancer Immunotherapy}

In the long-lasting campaign against cancer, four general approaches have been developed: surgery, chemotherapy, radiotherapy, and most recently, immunotherapy, which has evolved into a promising cancer treatment modality (Floudas et al., 2019). Inspired by the victory achieved with ICIs in the last decade, oncologists and immunologists have made every endeavor to secure further success. However, the success achieved with ICIs is still limited, thus demanding more directions to be explored. The incidental discovery of anticancer effect induced by E. granulosus infection have gained much focus. Understanding the underling mechanisms may provide some new insights for immunotherapy orchestration.

\section{Echinococcal Molecules With Anticancer Potential}

Available evidences have led to a hypothesis that E. granulosus may induce anticancer effect through hydatid molecules, especially the protoscolex ES molecules. Although the specific molecules and the underlying mechanisms remain largely unknown, EgKI1 , a potent chymotrypsin and neutrophil elastase inhibitor highly expressed by E. granulosus (Ranasinghe et al., 2015), was able to induce anticancer effect both in vitro and in animal models (Ranasinghe et al., 2018). In addition to the potent inhibitory effect on neutrophil elastase, which plays a crucial role in cancer metastasis, EgKI-1 can also induce anticancer effect by directly inhibiting tumor growth, probably through disrupting cell cycle progression, thus increasing cancer cell apoptosis. Meanwhile, the relatively low molecular weight of EgKI-1 $(<10 \mathrm{kDa})$ may allow it to penetrate tumor tissues effectively, thus facilitating its interaction with malignant cells. Therefore, EgKI-1 seems to be a promising therapeutic molecule against cancer, which may be considered in future treatment development.

\section{Echinococcal Antigens as Cancer Vaccine for Immunotherapy}

Successful ICIs treatment depends not only on the expression of checkpoint protein but also the immunogenicity of the cancer cells, i.e., whether the tumor is "immune competent" or "immune deficient" (Kyi and Postow, 2016). "Immune competent" means that the immunogenicity of cancer cell is strong enough to induce immune response of infiltrating $\mathrm{T}$ cells, which are unable to function normally due to checkpoint blockade or other immunoregulatory mechanisms (Mougiakakos et al., 2010; Shields et al., 2010); in direct contrast, "immune deficient" means that the immunogenicity of cancer cell is not sufficient to be recognized, thus enabling tumor to escape from host immune surveillance even after the checkpoint blockade has been relieved by ICIs (Hanahan and Weinberg, 2011; Tumeh et al., 2014).

After near 40 years of extensive research with only limited success, it is now believed that cancer vaccine may have the potential to convert immune-deficient tumor amenable to ICIs treatment through activation of T cell immunity (Lippitz, 2013; Maeng and Berzofsky, 2019). In this context, an ideal cancer vaccine should be, first, able to stimulate strong Th-1 response specifically (since Th-2 response may facilitate cancer progression and metastasis), and second, tumor-specific (inducing adaptive immunity specifically against cancer cells while sparing normal cells). Many tumor-specific antigens have been identified and exploited. Most of them, however, cannot trigger proper and effective immune response (Buonaguro et al., 2010), including the tumor associated mucin-type O-glycans Tn, sTn, and T antigen (Fu et al., 2016), which are almost exclusively found in cancer cells and have been widely employed in cancer diagnosis and prognosis prediction (Kudelka et al., 2015). Notably, an sTn-based vaccine failed to benefit women with metastatic breast cancer despite high level of specific immunoglobulin $G$ production and immunoglobulin M-to-immunoglobulin G seroconversion in a phase III multicenter study (Miles et al., 2011). A Th-2 dominant response may be an explanation for the failure of this vaccine, thus highlighting the importance of a Th-1-dominant response.

As noted above, the mucin-type O-glycans have already been detected both in adult and larval stage of E. granulosus and in patients with CE (Alvarez Errico et al., 2001; Casaravilla et al., 2003). On the other hand, accumulating evidences have demonstrated that hydatic cyst antigens can induce anticancer effect against several cancer types in vitro and in murine models (Aref et al., 2013; Darani et al., 2016; Rostami et al., 2018; Darani et al., 2018). Furthermore, immunization of mice with echinococcal antigens, whether Tn-like peptide or crude hydatid cyst antigens, resulted in high level of IFN- $\gamma$ production, indicating a Th-1-polarized response (Noya et al., 2013). Taken together, these findings attempt us to hypothesize that the hydatid cyst antigens, especially the mucin like O-glycans, may induce anticancer effect through activation of Th-1-polarized immune response. Therefore, the mucin-like O-glycans of $E$. granulosus may be considered as potential candidate in future vaccine design. Since there are great differences between human and murine immune system, additional studies are required to verify this hypothesis.

\section{CONCLUSION}

Accumulating evidences have suggested that the canine tapeworm E. granulosus can induce anticancer effect against several cancer types in vitro and in murine model, presumably through activating Th-1-polarized immune response with common antigens, especially the mucin-type O-glycans, and secreting molecules with anticancer potential, EgKI-1 in particular. Hopefully, these findings may provide some new insights into immunotherapy and replenish our arsenal against cancer. With a new era of immunotherapy at dawn, further studies are required to shed further lights on the mechanism underling the Th- 1 response induced by mucin-type O-glycans and to identify additional ES products with anticancer potential. In addition to anticancer effect, several research groups have reported that $E$. granulosus could not only provide protective effect against Taenia multiceps infection in sheep (Gauci et al., 2008) but also against experimental colitis (Soufli et al., 2015; Khelifi et al., 2017) and airway inflammation (Wang et al., 2014; Kim et al, 2019). The exact mechanism underlying these protective effects remain largely unknown, possibly related to antigen similarity and 
immunomodulatory effect from echinococcal molecules, which are also worthy of further exploring.

\section{AUTHOR CONTRIBUTIONS}

GW and WX drafted this manuscript. GW designed the artwork and revised the manuscript according to reviewers' comments. LS and ZX refined English writing. WX, ZX, and ZJ reviewed this manuscript.

\section{REFERENCES}

Akgül, H., Tez, M., Unal, A. E., Keşkek, M., Sayek, I., and Ozçelik, T. (2003). Echinococcus against cancer: why not? Cancer. 98, 1999-2000. doi: 10.1002/ cncr.11752

Almeida, G. F. G., Sarinho, F. W., Carvalho de Abreu e Lima, P., Oliveira Filho, J. B., Moura, M. A., de, L., et al. (2017). DNA repair defect and RAS mutation in two patients with Schistosoma mansoni-associated colorectal cancer: carcinogenesis steps or mere coincidence? J. Glob. Oncol. 3, 423-426. doi: 10. 1200/JGO.2016.006254

Altun, A., Saraydin, S. U., Soylu, S., Inan, D. S., Yasti, C., Ozdenkaya, Y., et al. (2015). Chemopreventive effects of hydatid disease on experimental breast cancer. Asian. Pac. J. Cancer. Prev. 16, 1391-1395. doi: 10.7314/APJCP.2015.16.4.1391

Alvarez Errico, D., Medeiros, A., Míguez, M., Casaravilla, C., Malgor, R., Carmona, C., et al. (2001). O-Glycosylation in Echinococcus granulosus: identification and characterization of the carcinoma-associated Tn antigen. Exp. Parasitol. 98, 100-109. doi: 10.1006/expr.2001.4620

Aref, N., Shirzad, H., Yousefi, M., and Darani, H. Y. (2013). Effect of different hydatid cyst molecules on hela and vero cell lines growth in vitro. J. Immunodefic. Disor. 2, 1. doi: 10.4172/2324-853X.1000105

Balar, A. V., and Weber, J. S. (2017). PD-1 and PD-L1 antibodies in cancer: current status and future directions. Cancer. Immunol. Immunother. 66, 551-564. doi: 10.1007/s00262-017-1954-6

Benchekroun, A., Lakrissa, A., Mikou, A., and Benosmane, A. (1979). Association of renal sarcoma with hydatid cyst of the kidney (apropos of a case). Maroc. Med. 1, 343-347. doi: 10.1016/j.ceramint.2006.02.010

Berriel, E., Russo, S., Monin, L., Festari, M. F., Berois, N., Fernández, G., et al. (2013). Antitumor activity of human hydatid cyst fluid in a murine model of colon cancer. Sci. World. J. 2013, 1-7. doi: 10.1155/2013/230176

Berry, A., Iriart, X., Fillaux, J., and Magnaval, J. F. (2017). Urinary schistosomiasis and cancer. Bull. Soc. Pathol. Exot. 110, 68-75. doi: 10.1007/s13149-017-0547-4

Bourne, M. S., and Williams, G. E. (1963). Compression of the inferior vena cava by hydatid disease of the liver, with associated hepatocellular carcinoma. JAMA. 186, 235. doi: 10.1001/jama.1963.03710070159097

Brunetti, E., Kern, P., and Vuitton, D. A. (2010). Expert consensus for the diagnosis and treatment of cystic and alveolar echinococcosis in humans. Acta. Trop. 114, 1-16. doi: 10.1016/j.actatropica.2009.11.001

Buonaguro, L., Petrizzo, A., Tornesello, M. L., and Buonaguro, F. M. (2010). Translating tumor antigens into cancer vaccines. Clin. Vaccine. Immunol. 18, 23-34. doi: 10.1128/CVI.00286-10

Callejas, B. E., Martínez Saucedo, D., and Terrazas, L. I. (2018). Parasites as negative regulators of cancer. Biosci. Rep. 38, 1-7. doi: 10.1042/BSR20180935

Casaravilla, C., Malgor, R., and Carmona, C. (2003). Characterization of carbohydrates of adult Echinococcus granulosus by lectin-binding analysis. J. Parasitol. 89, 57-61. doi: 10.1645/0022-3395(2003)089[0057:COCOAE]2.0 $\mathrm{CO} ; 2$

Champiat, S., Lambotte, O., Barreau, E., Belkhir, R., Berdelou, A., Carbonnel, F., et al. (2015). Management of immune checkpoint blockade dysimmune toxicities: a collaborative position paper. Ann. Oncol. 27, 559-574. doi: 10.1093/ annonc/mdv623

Chookami, B. M., Sharafi, S. M., Rafeie, R., Bahadoran, M., Pestechian, N., and Darani, H. Y. (2014). Effect of alive protoscoleces of hydatid cyst on the growth of melanoma cells in mouse model. J. Isfahan. Med. School. 32, 486-492.

Daneshpour, S., Bahadoran, M., Hejazi, S. H., Eskandarian, A. A., Mahmoudzadeh, M., and Darani, H. Y. (2016). Common antigens between hydatid cyst and cancers. Adv. Biomed. Res. 5, 9. doi: 10.4103/2277-9175.175242

\section{FUNDING}

The publication of this review has been funded by A Major Infectious Disease Prevention and Control of the National Science and Technique Major Project (NO. 2018ZX10715004), Project from Department of Education of Sichuan (NO. 18ZB0241) and Research Project from Sichuan Health and Family Planning Commission (NO.16PJ511).

Daneshpour, S., Kefayat, A. H., Mofid, M. R., Rostami, R. S., and Darani, H. Y. (2019). Effect of hydatid cyst fluid antigens on induction of apoptosis on breast cancer cells. Adv. Biomed. Res. 8, 27. doi: 10.4103/abr.abr_220_18

Darani, H. Y., Soozangar, N., Khorami, S., Taji, F., Yousofi, M., and Shirzad, H. (2012). Hydatid cyst protoscolices induce cell death in WEHI-164 fibrosarcoma cells and inhibit the proliferation of baby hamster kidney fibroblasts in vitro. J. Parasitol. Res. 2012, 304183. doi: 10.1155/2012/304183

Darani, H. Y., and Yousefi, M. (2012). Parasites and cancers: parasite antigens as possible targets for cancer immunotherapy. Future Oncol. 8, 1529-1535. doi: $10.2217 /$ fon. 12.155

Darani, H. Y., Sharafi, S. M., Mokarian, M., Yousefi, M., Sharafi, S. A., and Jafari, R. (2016). Therapeutic effect of hydatid cyst liquid on melanoma tumor growth in mouse model. Br. J. Med. Med. Res. 18, 27220. doi: 10.9734/BJMMR/2016/27220

Darani, H. Y., Nodeh, F. J., Ramazaninia, S. T., and Sharafi., S. M. (2018). Effect of immune responses against hydatid cyst antigens on growth of melanoma tumor. Immunoregulation 1, 107-112. doi: 10.32598/ IMMUNOREGULATION.1.2.101

Darvin, P., Toor, S. M., Sasidharan Nair, V., and Elkord, E. (2018). Immune checkpoint inhibitors: recent progress and potential biomarkers. Exp. Mol. Med. 50, 165. doi: 10.1038/s12276-018-0191-1

Didierlaurent, A. M., Morel, S., Lockman, L., Giannini, S. L., Bisteau, M., Carlsen, H., et al. (2009). AS04, an aluminum salt- and TLR4 agonist-based adjuvant system, induces a transient localized innate immune response leading to enhanced adaptive immunity. J. Immunol. 183, 6186-6197. doi: 10.4049/ jimmunol.0901474

Feng, M., and Cheng, X. (2017). Parasite-associated cancers (blood flukes/liver flukes). Adv. Exp. Med. Biol. 1018, 193-205. doi: 10.1007/978-981-10-5765-6_12

Floudas, C. S., Brar, G., and Greten, T. (2019). Immunotherapy: current status and future perspectives. Dig. Dis. Sci. 64, 1030-1040. doi: 10.1007/ s10620-019-05516-7

Fu, C., Zhao, H., Wang, Y., Cai, H., Xiao, Y., Zeng, Y., et al. (2016). Tumorassociated antigens: Tn antigen, sTn antigen, and T antigen. HLA. 88, 275-286. doi: $10.1111 / \tan .12900$

Gauci, C., Vural, G., Öncel, T., Varcasia, A., Damian, V., Kyngdon, C. T., et al. (2008). Vaccination with recombinant oncosphere antigens reduces the susceptibility of sheep to infection with Taenia multiceps. Int. J. Parasitol. 38, 1041-1050. doi: 10.1016/j.ijpara.2007.11.006

Gottstein, B., Soboslay, P., Ortona, E., Wang, J., Siracusano, A., and Vuitton, D. A. (2017). Immunology of alveolar and cystic echinococcosis (AE and CE). Adv. Parasitol. 96, 1-54. doi: 10.1016/bs.apar.2016.09.005

Gungor, T., Altinkaya, S. O., Sirvan, L., Lafuente, R. A., and Ceylaner, S. (2011). Coexistence of borderline ovarian epithelial tumor, primary pelvic hydatid cyst, and lymphoepithelioma-like gastric carcinoma. Taiwan. J. Obstet. Gynecol. 50, 201-204. doi: 10.1016/j.tjog.2009.10.005

Hamid, H. K. S. (2019). Schistosoma japonicum-associated colorectal cancer: a review. Am. J. Trop. Med. Hyg. 100, 501-505. doi: 10.4269/ajtmh.18-0807

Hanahan, D., and Weinberg, R. A. (2011). Hallmarks of cancer: the next generation. Cell. 144, 646-674. doi: 10.1016/j.cell.2011.02.013

Kallinikova, V. D., Batmonkh, T., Kosobokova, E. N., Pakhorukova, L. V., and Matekin, P. V. (2008). Antibodies against Trypanosoma cruzi in intact mice and their oncoprotective effect. Med. Parazitol (Mosk). 1, 11-15.

Kang, Y. J., Jo, J. O., Cho, M. K., Yu, H. S., Leem, S. H., Song, K. S., et al. (2013). Trichinella spiralis infection reduces tumor growth and metastasis of B16-F10 melanoma cells. Vet. Parasitol. 196, 106-113. doi: 10.1016/j. vetpar.2013.02.021 
Karadayi, S., Arslan, S., Sumer, Z., Turan, M., Sumer, H., and Karadayi, K. (2013). Does hydatid disease have protective effects against lung cancer? Mol. Biol. Rep. 40, 4701-4704. doi: 10.1007/s11033-013-2565-8

Kudelka, M. R., Ju, T., Heimburg-Molinaro, J., and Cummings, R. D. (2015). Simple sugars to complex disease-mucin-type O-glycans in cancer. Adv. Cancer. Res. 126, 53-135. doi: 10.1016/bs.acr.2014.11.002

Khelifi, L., Soufli, I., Labsi, M., and Touil-Boukoffa, C. (2017). Immune-protective effect of echinococcosis on colitis experimental model is dependent of down regulation of TNF- $\alpha$ and NO production. Acta. Trop. 166, 7-15. doi: 10.1016/j. actatropica.2016.10.020

Kim, H. J., Kang, S. A., Yong, T. S., Shin, M. H., Lee, K. J., Park, G. M., et al. (2019). Therapeutic effects of Echinococcus granulosus cystic fluid on allergic airway inflammation. Exp. Parasitol. 198, 63-70. doi: 10.1016/j.exppara.2019.02.003

Kyi, C., and Postow, M. A. (2016). Immune checkpoint inhibitor combinations in solid tumors: opportunities and challenges. Immunotherapy 8, 821-837. doi: 10.2217/imt-2016-0002

Li, H., Song, T., Shao, Y., and Wen, H. (2015). Cystic echinococcosis accompanied by hepatocellular carcinoma in a female herdsman. Int. J. Clin. Exp. Med. 8, 2985-2988.

Lippitz, B. E. (2013). Cytokine patterns in patients with cancer: a systematic review. Lancet Oncol. 14, e218-e228. doi: 10.1016/S1470-2045(12)70582-X

Maeng, H. M., and Berzofsky, J. A. (2019). Strategies for developing and optimizing cancer vaccines. F1000Res. 8, 654. doi: 10.12688/f1000research.18693.1

Meichenin, M., Rocher, J., Galanina, O., Bovin, N., Nifantev, N., Sherman, A., etal. (2000). $\mathrm{Tk}$, a new colon tumor-associated antigen resulting from altered o-glycosylation. Cancer. Res. 60, 5499-5507. doi: 10.1097/00002820-200010000-00012

Miles, D., Roche, H., Martin, M., Perren, T. J., Cameron, D. A., Glaspy, J., et al. (2011). Phase III multicenter clinical trial of the sialyl-TN (STn)-keyhole limpet hemocyanin $(\mathrm{KLH})$ vaccine for metastatic breast cancer. Oncologist. 16, 1092-1100. doi: 10.1634/theoncologist.2010-0307

Miñana López, B., Fernández Aparicio, T., Vazquez, B. S., Carrero López, V., Rodríguez Antolin., A., Passas Martínez, J., et al. (1994). Hydatid cyst and renal adenocarcinoma: an exceptional association. Actas. Urol. Esp. 18, 58-63.

Misthos, P., Kanakis, M. A., Horti, M., Kakaris, S., and Lioulias, A. (2012). Coexistence of a lung carcinosarcoma and hydatid cyst. Updates. Surg. 65, 253-254. doi: 10.1007/s13304-012-0145-4

Mougiakakos, D., Choudhury, A., Lladser, A., Kiessling, R. and Johansson, C. C., et al. (2010). Regulatory T cells in cancer. Adv. Cancer. Res. 107, 57-117. doi: 10.1016/S0065-230X(10)07003-X

Muralidhar, V., Santhaseelan, R. G., Ahmed, M., and Shanmuga, P. (2018). Simultaneous occurrence of hepatic hydatid cyst and mucinous cystadenoma of the liver in a middle-aged female patient: report of a rare case. BMJ Case Rep. 2018, 1-5 .doi: 10.1136/bcr-2018-226077

Noya, V., Bay, S., Festari, M. F., García, E. P., Rodriguez, E., Chiale, C., et al. (2013). Mucin-like peptides from Echinococcus granulosus induce antitumor activity. Int. J. Oncol. 43, 775-784. doi: 10.3892/ijo.2013.2000

Nyame, K., Cummings, R. D., and Damian, R. T. (1987). Schistosoma mansoni synthesizes glycoproteins containing terminal o-linked n-acetylglucosamine residues. J. Biol. Chem. 262, 7990-7995.

Oikonomopoulou, K., Yu, H., Wang, Z., Vasiliou, S. K., Brinc, D., Christofi, G., et al. (2016). Association between Echinococcus granulosus infection and cancer risk-a pilot study in Cyprus. Clin. Chem. Lab. Med. 54, 1955-1961. doi: $10.1515 / \mathrm{cclm}-2016-0125$

Osinaga, E. (2007). Expression of cancer-associated simple mucin-type O-glycosylated antigens in parasites. IUBMB. Life. 59, 269-273. doi: $10.1080 / 15216540601188553$

Ramazninia, S. T., Sharafi, S. M., Bahadoran, M., Nodeh., F. J., Mahmoudzadeh, M., and Darani, H. Y. (2016). Effect of passive transfer of spleen cells from immunized mice with hydatid cyst antigens on the growth of melanoma cancer in C57/black mice. BJMMR. 16, 1-6. doi: 10.9734/BJMMR/2016/26744

Ranasinghe, S. L., Fischer, K., Zhang, W., Gobert, G. N., and McManus, D. P. (2015). Cloning and characterization of two potent kunitz type protease inhibitors from Echinococcus granulosus. PLoS. Negl. Trop. Dis. 9, e0004268. doi: 10.1371/journal.pntd.0004268

Ranasinghe, S. L., Boyle, G. M., Fischer, K., Potriquet, J., Mulvenna, J. P., and McManus, D. P. (2018). Kunitz type protease inhibitor EgKI-1 from the canine tapeworm Echinococcus granulosus as a promising therapeutic against breast cancer. PLoS One 13, e0200433. doi: 10.1371/journal.pone.0200433
Ranasinghe, S. L., and McManus, D. P. (2018). Echinococcus granulosus: cure for cancer revisited. Front. Med. (Lausanne). 5, 60. doi: 10.3389/ fmed.2018.00060

Savoia, P., Astrua, C., and Fava, P. (2016). Ipilimumab (Anti-Ctla-4 Mab) in the treatment of metastatic melanoma: effectiveness and toxicity management. Hum. Vaccin. Immunother. 12, 1092-1101. doi: 10.1080/ 21645515.2015.1129478

Sharafi, S. M., Rafiei, R., Hadipour, M., Shirzad, H., Khanahmad, H., and Darani, H. F. (2016). A nonglycosylated $27 \mathrm{kDa}$ molecule as common antigen between human breast cancer and Echinococcus granulosus hydatid cyst wall. Adv. Breast. Cancer. Res. 5, 90-95. doi: 10.4236/abcr.2016.52010

Sharafi, S. M., Shirzad, H., Khanahmad, H., Ataei, B., and Darani, H. Y. (2017). Monoclonal antibodies production against a $40 \mathrm{kDa}$ band of hydatid cyst fluid. Recent. Pat. Biotechnol. 12 (1), 90-95. doi: 10.2174/18722083116661 70317151346

Shields, J. D., Kourtis, I. C., Tomei, A. A., Roberts, J. M., and Swartz, M. A. (2010). Induction of lymphoid-like stroma and immune escape by tumors that express the chemokine CCL21. Science 328, 749-752. doi: 10.1126/science.1185837

Shurin, M. R., Lu, L., Kalinski, P., Stewart-Akers, A. M., and Lotze, M. T. (1999). Th1/Th2 balance in cancer, transplantation and pregnancy. Springer Semin. Immunopathol. 21, 339-359. doi: 10.1007/BF00812261

Soufli, I., Toumi, R., Rafa, H., Amri, M., Labsi, M., Khelifi, L., et al. (2015). Crude extract of hydatid laminated layer from Echinococcus granulosus cyst attenuates mucosal intestinal damage and inflammatory responses in dextran sulfate sodium induced colitis in mice. J. Inflamm. 12 (1), 1-12. doi: 10.1186/s12950-015-0063-6

Rostami, S. R., Daneshpour, S., Mofid, M. R., Andalib, A., Eskandariyan, A., and Darani, H. Y. (2018). Effect of hydatid cyst antigens on inhibition of melanoma cancer growth in mouse model. Cell. Mol. Biol. 64, 1-5. doi: 10.14715/ $\mathrm{cmb} / 2018.64 .12 .1$

Takemura, Y., Kikuchi, S., and Inaba, Y. (1998). Epidemiologic study of the relationship between schistosomiasis due to Schistosoma japonicum and liver cancer/cirrhosis. Am. J. Trop. Med. Hyg. 59, 551-556. doi: 10.4269/ ajtmh.1998.59.551

Tez, S., and Tez, M. (2015). Echinococcus and cancer: unsolved mystery. Parasite. Immunol. 37, 426-426. doi: 10.1111/pim.12201

Trinchieri, G. (2015). Cancer immunity: lessons from infectious diseases. J. Infect. Dis. 212, S67-S73. doi: 10.1093/infdis/jiv070

Tumeh, P. C., Harview, C. L., Yearley, J. H., Shintaku, I. P., Taylor, E. J. M., Robert, L. (2014). PD-1 blockade induces responses by inhibiting adaptive immune resistance. Nature 515, 568-571. doi: 10.1038/nature13954

Turhan, N., Esendagli, G., Ozkayar, O., Tunali, G., Sokmensuer, C., and Abbasoglu, O. (2015). Co-existence of Echinococcus granulosus infection and cancer metastasis in the liver correlates with reduced Th1 immune responses. Parasite. Immunol. 37, 16-22. doi: 10.1111/pim.12152

Ubillos, L., Medeiros, A., Cancela, M., Casaravilla, C., Saldaña, J., Domínguez, L., et al. (2007). Characterization of the carcinoma-associated Tk antigen in helminth parasites. Exp. Parasitol. 116, 129-136. doi: 10.1016/j.exppara.2006.12.009

Wang, H., Li, J., Pu, H., Hasan, B., Ma, J., Jones, M. K., et al. (2014). Echinococcus granulosus infection reduces airway inflammation of mice likely through enhancing IL-10 and down-regulation of IL-5 and IL-17A. Parasit. Vectors. 7 (1), 1-3. doi: 10.1186/s13071-014-0522-6

Wen, H., Vuitton, L., Tuxun, T., Li, J., Vuitton, D. A., Zhang, W., et al. (2019). Echinococcosis: advances in the 21st century. Clin. Microbiol. Rev. 32 (2), 1-39. doi: 10.1128/CMR.00075-18

Yong, W. K., Heath, D. D., and Savage, T. (1979). Possible antigenic similarity between pulmonary carcinoma and cysts of Echinococcus granulosus. BMJ 1, 1463-1464. doi: 10.1136/bmj.1.6176.1463-a

Zamarron, B. F., and Chen, W. (2011). Dual roles of immune cells and their factors in cancer development and progression. Int. J. Bioll. Sci. 7, 651-658. doi: 10.7150/ijbs.7.651

Zenina, A. V., Kravtsov, E. G., Tsetsegsaikhan, B., Yashina, N. V., Dalin, M. V., Karpenko, L. P., et al. (2008). The study of immunological component in antitumor effect of Trypanosoma cruzi. Bull. Exp. Biol. Med. 145, 352-354. doi: 10.1007/s10517-008-0089-3

Zhang, W., Ross, A. G., and McManus, D. P. (2008). Mechanisms of immunity in hydatid disease: implications for vaccine development. J. Immunol. 181, 66796685. doi: 10.4049/jimmunol.181.10.6679 
Zhang, X., Zhang, W., Yuan, X., Fu, M., Qian, H., and Xu, W. (2016). Neutrophils in cancer development and progression: roles, mechanisms, and implications (Review). Int. J. Oncol. 49, 857-867. doi: 10.3892/ijo.2016.3616

Zhigunova, A. V., Kravtsov, E. G., Yashina, N. V., Dalin, M. V., and Karpenko, L. P. (2013). Effects of specific antibodies and immunocompetent cells on tumor growth in passive transfer experiment. Bull. Exp. Biol. Med. 154 (6), 762-764. doi: 10.1007/s10517-013-2050-3

Zöld, É., Barta, Z., and Zeher, M. (2005). Hydatid disease of the liver and associated hepatocellular carcinoma. Clin. Gastroenterol. Hepatol. 3, A35-A35. doi: 10.1016/S1542-3565(05)00288-0
Conflict of Interest: The authors declare that the research was conducted in the absence of any commercial or financial relationships that could be construed as a potential conflict of interest.

Copyright $(2019$ Guan, Zhang, Wang, Lu, Yin and Zhang. This is an open-access article distributed under the terms of the Creative Commons Attribution License (CC BY). The use, distribution or reproduction in other forums is permitted, provided the original author(s) and the copyright owner(s) are credited and that the original publication in this journal is cited, in accordance with accepted academic practice. No use, distribution or reproduction is permitted which does not comply with these terms. 